\title{
ARTICLE OPEN Protein activation mapping of human sun-protected epidermis after an acute dose of erythemic solar simulated light
}

Janine G. Einspahr ${ }^{1,2}$, Clara Curiel-Lewandrowski ${ }^{1,2}$, Valerie S. Calvert ${ }^{3}$, Steven P. Stratton ${ }^{1,2}$, David S. Alberts ${ }^{1,2}$, James Warneke ${ }^{1,4}$, Chengcheng $\mathrm{Hu}^{5}$, Kathylynn Saboda ${ }^{1}$, Elisabeth L. Wagener ${ }^{1}$, Sally Dickinson ${ }^{1,2}$, Zigang Dong ${ }^{6}$, Ann M. Bode ${ }^{6}$ and

Emanuel F. PetricoinlII ${ }^{3}$

Ultraviolet radiation is an important etiologic factor in skin cancer and a better understanding of how solar stimulated light (SSL) affects signal transduction pathways in human skin which is needed in further understanding activated networks that could be targeted for skin cancer prevention. We utilized Reverse Phase Protein Microarray Analysis (RPPA), a powerful technology that allows for broad-scale and quantitative measurement of the activation/phosphorylation state of hundreds of key signaling proteins and protein pathways in sun-protected skin after an acute dose of two minimal erythema dose (MED) of SSL. RPPA analysis was used to map the altered cell signaling networks resulting from acute doses of solar simulated radiation (SSL). To that end, we exposed sun-protected skin in volunteers to acute doses of two MED of SSL and collected biopsies pre-SSL and post-SSL irradiation. Frozen biopsies were subjected to laser capture microdissection (LCM) and then assessed by RPPA. The activation/phosphorylation or total levels of 128 key signaling proteins and drug targets were selected for statistical analysis. Coordinate network-based analysis was performed on specific signaling pathways that included the PI3k/Akt/mTOR and Ras/Raf/MEK/ERK pathways. Overall, we found early and sustained activation of the PI3K-AKT-mTOR and MAPK pathways. Cell death and apoptosis-related proteins were activated at 5 and $24 \mathrm{~h}$. Ultimately, expression profile patterns of phosphorylated proteins in the epidermal growth factor receptor (EGFR), AKT, mTOR, and other relevant pathways may be used to determine pharmacodynamic activity of new and selective topical chemoprevention agents administered in a test area exposed to SSL to determine drug-induced attenuation or reversal of skin carcinogenesis pathways.

npj Precision Oncology (2017)1:34 ; doi:10.1038/s41698-017-0037-7

\section{INTRODUCTION}

Skin cancer, which includes both nonmelanoma skin cancers (NMSC) and melanoma, is the most common malignancy in the United States and its incidence is rapidly increasing. ${ }^{1,2}$ Skin cancer represents a significant public health and economic burden estimated to be over 8.1 billion dollars annually ${ }^{3}$ and consists of $75-80 \%$ basal cell carcinomas (BCC) and approximately $18 \%$ cutaneous squamous cell carcinomas (SCC). This high incidence of skin cancer can be directly related to chronic solar radiation exposure. ${ }^{4}$ Since the primary etiologic agent for cutaneous SCC is solar radiation, it should be possible to reduce the risk of cutaneous SCCs using current sun avoidance methods. ${ }^{4}$ Unfortunately, these current prevention strategies, which include sun avoidance and ultraviolet (UV) protection, have shown limited success. ${ }^{5}$ Therefore, there is a need to understand the molecular alterations that take place and to develop new prevention strategies to reduce the risk of skin cancers.

UV radiation is a complete carcinogen meaning that UV exposure stimulates initiation, promotion, and progression to cutaneous SCCs. ${ }^{6-8}$ As an initiator, UV radiation results in genetic mutations, many of which are signature UV mutations in critical genes. Additionally, UV can act as a promoter through the activation of multiple signal transduction pathways. ${ }^{9}$ The UV spectrum reaching the earth's surface is 90-95\% UVA (320-400 $\mathrm{nm})$ and $5-10 \%$ UVB $(280-320 \mathrm{~nm}) .{ }^{10}$ Many studies of UV-induced skin carcinogenesis have focused on UVB, which has been most closely linked to DNA damage while UVA has been associated more with skin cancer promotion. UVA and UVB have primarily been studied separately with regard to the activation of signaling pathways and skin carcinogenesis. ${ }^{1-15}$ Fewer studies have focused on the entire spectrum of UVA and UVB combined in a ratio that closely mimics the solar spectrum. ${ }^{16}$ Strong experimental evidence indicates that exposure of epidermal cells to UV results in the activation of numerous signal transduction pathways that include the PI3K/Akt-mTOR and MAPK cascades, ${ }^{10}$ with an effect that appears to be both time and wavelength dependent. ${ }^{17}$

In this study we utilized Reverse phase protein array (RPPA), a novel platform that allows for the broad-scale and quantitative measurement of the activation/phosphorylation state of dozens to hundreds of proteins to interrogate the activation of total proteins and phosphoproteins in usually sun-protected skin after an acute dose of two MED of solar stimulated light (SSL). RPPA is a powerful

\footnotetext{
${ }^{1}$ The University of Arizona Cancer Center, 1515 North Campbell Ave., PO Box 245024, Tucson, AZ, USA; ${ }^{2}$ Department of Medicine, The University of Arizona, Tucson, AZ, USA; ${ }^{3}$ Center for Applied Proteomics and Molecular Medicine, George Mason University, Manassas, VA, USA; ${ }^{4}$ Department of Surgery, The University of Arizona, Tucson, AZ, USA; ${ }^{5}$ Department of Epidemiology and Biostatistics, The University of Arizona, Tucson, AZ, USA and ${ }^{6}$ Department of Molecular Medicine and Biopharmaceutical Sciences, The Hormel Institute, The University of Minnesota, Austin, MN, USA

Correspondence: Clara Curiel-Lewandrowski (ccuriel@email.arizona.edu)

Janine G. Einspahr and Clara Curiel-Lewandrowski contributed equally to this work.
}

Received: 5 December 2016 Revised: 19 June 2017 Accepted: 15 August 2017

Published online: 21 September 2017 
technology that provides a means to measure large number of ultra-low abundance signaling proteins from small amounts of input material including LCM clinical samples. Gene expression arrays are commonly utilized to measure changes in the mRNA expression, but the measurement of RNA alone could potentially miss critical information about protein drug target levels as well as post-translational modifications, such as protein phosphorylation, which drive cellular processes and control cell signaling. ${ }^{18}$ Pathways included the PI3k/Akt/mTOR and Ras/Raf/MEK/ERK pathways.

\section{RESULTS}

We exposed normally sun-protected human buttock skin to a single acute dose of two MED SSL and obtained snap frozen specimens pre- and post-SSL in 12 healthy subjects with similar types of skin (Fitzpatrick skin type II or III). The study included 8 males and 4 females [average age of $67.6 \pm 10.6$ (mean \pm standard deviation) years for males and $66.2 \pm 6.6$ years for females]. All subjects were Caucasian and non-Hispanic. Fifty-eight percent (7/ 12) of the subjects had Fitzpatrick skin Type II (usually burns, tans less than average, with difficulty) while $42 \%$ (5/12) had skin Type III (sometimes mild burn, tans about average). ${ }^{19}$ The solar simulator delivered $8.7 \%$ of UVB and $91.3 \%$ of UVA $^{19}$ and the average ( \pm standard error) dose delivered was $3.6 \pm 0.5 \mathrm{~J} / \mathrm{cm}^{2}$ of UVA and $51.6 \pm 7.0 \mathrm{~mJ} / \mathrm{cm}^{2}$ of UVB.

Biopsies were split with $1 / 2$ in formalin for immunohistochemistry $(\mathrm{IHC})^{20}$ and $1 / 2$ snap frozen for RPPA. Samples underwent LCM to enrich samples for epidermal cells, followed by sample lysis, and printing onto RPPA slides. RPPA slides were stained with antibodies that have been extensively pre-validated for RPPA by single band Western blotting and peptide competition. The analysis yielded data for 128 proteins/phosphoproteins, which were chosen based on literature and knowledge of signaling pathway alterations seen in both mouse and human skin after UV irradiation and in the progression of normal skin to cutaneous SCC. When the four time points post-SSL ( $5 \mathrm{~min}, 1,5$, and $24 \mathrm{~h}$ ) were compared to baseline (no SSL), 42 of these investigated proteins had a $p$-value of $\leq 0.05$, but due to the large number of comparisons a $p$-value of $\leq 0.0125$ was required to be considered statistically significant after adjusting for multiple comparisons. This analytical process yield 33 proteins with a $p$-value of $\leq 0.0125$ at any of the time points. There were four proteins with marginal significance $(p \leq<0.05)$ in any of the time points, but the Trend Test was statistically significant $(p \leq 0.0125)$. In addition, there were 15 proteins where only the Trend Test was significant ( 8 at $p \leq 0.0125$ and 7 at $p \leq 0.05$ ).

Table 1 shows the $p$-values for each time point following two MEDs of SSL ( $5 \mathrm{~min}, 1 \mathrm{~h}, 5 \mathrm{~h}$, and $24 \mathrm{~h}$ ) compared to baseline (no SSL). Table 1 also shows $p$-values for trend. All of the Cancer Landscape (CScape) Protein Pathway Activation Maps (example shown in Fig. 1) are available as Supplemental Figure S1). Overall, we saw activation of MAPK, AKT-mTOR and IGFR pathways, as well as the cell death/apoptosis-related pathways. Within the PI3 kinase/Akt signaling pathway, there were statistically significant results for phospho-ErbB3 (Y1289) at $1 \mathrm{~h}(p<0.001)$ and $5 \mathrm{~h}$ $(p<0.001)$ when compared to baseline levels, for phospho-IRS1 (S612) at $24 \mathrm{~h}(p=0.003)$, and for phospho-Akt (S473) at $5 \mathrm{~h}(p=$ $0.009)$ and $24 \mathrm{~h}(p=0.006)$. In Fig. 1 , the center, top panel compares the 5-min time point to baseline ( $A$, top center), and the 24-hr comparison to baseline is shown in the center bottom panel (B). The protein network maps indicate that there was very little SSL-induced signaling by $5 \mathrm{~min}$, whereas at $24 \mathrm{~h}$, many pathways were activated. In the CScape maps, significant positive differences are demarcated (balloons) in increasing shades of red, whereas higher negative differences are shown in increasing shades of green. White balloons represent no significant change. Each balloon pin is placed over each protein that was measured.
Magnified views of the PI3K/AKT/mTOR pathway (Fig. 1). Shown in the expanded CScape view are expression of phospho-mTOR (S2448), which was significantly increased at $5 \mathrm{~h}(p=0.005)$ and 24 h $(p<0.001)$, phospho-p70 S6 Kinase (S371) with borderline significance at $5 \mathrm{~h}(p=0.02)$ and significance at $24 \mathrm{~h}(p<0.001)$, phospho-elF4G (S1108) with borderline significance at $5 \mathrm{~h}(p=$ $0.02)$ and significant at $24 \mathrm{~h}(p<0.001)$. phospho-GSK-3a/B $(\mathrm{S} 21 / 9)$ was significantly different at $5 \mathrm{~h}(p=0.002)$ and $24 \mathrm{~h}(p=0.001)$, expression of p21 Waf1/Cip1 was significant at $24 \mathrm{~h}(p=0.001)$, p27 Kip1 $(p=0.012)$ was significant at $24 \mathrm{~h}$, phospho-PAK1/2 (T423/402) was significant at $24 \mathrm{~h}(p=0.009)$, phospho-MDM2 was significantly different at (S166) at $5 \mathrm{~h}(p<0.001)$ and $24 \mathrm{~h}(p<$ $0.001)$, phospho-Cyclin D1 (T286) was significant at $24 \mathrm{~h}(p=0.01)$. Phospho-eNOS (S1177) was statistically significant at $1 \mathrm{~h}(p=$ $0.009), 5 \mathrm{~h}(p=0.001)$, and $24 \mathrm{~h}(p=0.0004)$, and phospho-eNOS/ NOS III (S116) was significant at $24 \mathrm{~h}(p=0.02)$. Phospho-LKB1 (S428) was significant at $5 \mathrm{~h}(p=0.003)$ and $24 \mathrm{~h}(p<0.001)$. Expression of phospho-MDM2 (S166) was significant at $5 \mathrm{~h}(p=$ $0.007)$ and at $24 \mathrm{~h}(p=0.0005)$.

Within the MAPK pathways (Table 1), phospho-epidermal growth factor receptor (EGFR) (Y1068) was borderline significant at $1 \mathrm{~h} \quad(p=0.02)$ and statistically significant at $5 \mathrm{~h}(p=0.004)$. Phospho-EGFR Y1173 had borderline significance at $24 \mathrm{~h}(p=$ 0.042). Figure 1 also shows CScape maps focusing on the Ras/Raf/ MEK/ERK pathways [blue boxes with left top showing the $5 \mathrm{~min}$ time point (A1) and bottom left showing the $24 \mathrm{~h}$ time, (B1)]. Phospho-Shc (Y317) was borderline at $5 \mathrm{~h}(p=0.03)$, phospho-Src Family (Y416) was statistically significant at $1 \mathrm{~h}(p=0.007)$, and phospho-Src (Y527) had borderline significance at $24 \mathrm{~h}(p=0.02)$. For phospho-B-Raf (S445), results were borderline at $24 \mathrm{~h}(p=0.06)$, but the Trend Test $p$-value was significant $(p=0.003)$. PhosphMEK1/2 (S217/221) was significant at $24 \mathrm{~h}(p=0.002)$ while phospho-ERK1/2 (T202/Y20) was significant at $1 \mathrm{~h} \quad(p=0.009)$, borderline at $5 \mathrm{~h}(p=0.03)$, and significant at $24 \mathrm{~h}(p=0.005)$. Similar to the results for B-Raf, phospho-SAPK/JNK (T183/Y185) had borderline significance at $5 \mathrm{~h}(p=0.02)$ and $24 \mathrm{~h}(p=0.04)$, but the Trend Test was statistically significant $(p=0.003)$. Phospho-p38 MAPK (T180/Y182) was borderline at $1 \mathrm{~h}(p=0.02)$ but significant at $5 \mathrm{~h}(p=0.001)$ and $24 \mathrm{~h}(p=0.002)$. phospho-RSK3 (T356/S360) was significant at $5 \mathrm{~h}(p=0.009)$ and $24 \mathrm{~h}(p=0.005)$, Phospho-p90RSK (S380) was significant at $24 \mathrm{~h}(p<0.001)$, phospho-p90RSK (T359/ S363) was significant at $5 \mathrm{~h}(p=0.012)$ and $24 \mathrm{~h}(p=0.002)$, phospho-CREB (S133) was borderline at $1 \mathrm{~h}(p=0.03)$ and statistically significant at $5 \mathrm{~h}(p=0.001)$ and $24 \mathrm{~h}(p=0.002)$, Phospho-ATF-2 (T71) was borderline at $1 \mathrm{~h}(p=0.02)$, while at $5 \mathrm{~h}$ $(p=0.009)$ and $24 \mathrm{~h}(p=0.004)$ was statistically significant compared to baseline. Phospho-pHistone H3 (S10) showed a nonsignificant increase at $24 \mathrm{~h}(p=0.06$, Table 1$)$.

Within the apoptotic pathway, Bim had borderline significance when compared to baseline at 24-hrs $(p=0.03)$ and had a significant Trend Test $(p=0.005)$, phospho-BAD (S112) was statically significant at $1-\mathrm{hr}(p=0.009)$, at $5 \mathrm{~h}(p=0.005)$, and 24 h $(p<0.001)$. phospho-BAD (S155) was also statically significant at $5 \mathrm{~h}(p=0.003)$ and 24-hrs $(p=0.007)$ while phospho-FADD (S194) was borderline at $5 \mathrm{~h}(p=0.03)$ and significant at $24 \mathrm{~h}(p=0.001)$. Phospho-ATP-Citrate Lyase (S454) was significant at $5 \mathrm{~h}(p=$ $0.0004)$ and at $24 \mathrm{~h}(p=0.0005)$. Smac/Diablo was statically significant compared to baseline at $24 \mathrm{~h}(p=0.002)$. Cleaved caspase 6 was borderline at $24 \mathrm{~h}(p=0.02$, Table 1$)$

Additional proteins showing statistically significant results were phospho-Stat $3(\mathrm{~S} 727)$ at $24 \mathrm{~h}(p<0.001)$, phospho-anaplastic lymphoma kinase (ALK) (Y1604) was significant at $5 \mathrm{~h}$ and $24 \mathrm{~h}(p$ $=0.009$ ). Phospho-Ezrin/Radixin/Moesin T567/564/558 was significant at $5 \mathrm{~h}(p=0.012)$, and phospho-VEGFR2 Y996 was increased at $1 \mathrm{~h}(p=0.006)$ and $5 \mathrm{~h}(p=0.01)$.

To further evaluate the relevance of the 33 identified analytes demonstrating a statistically significant change from baseline to $24 \mathrm{~h}$ in our study (Table 1 ), a validity assessment was performed by 
Table 1. $p$-values for each time point and trend following 2 MEDs of SSL

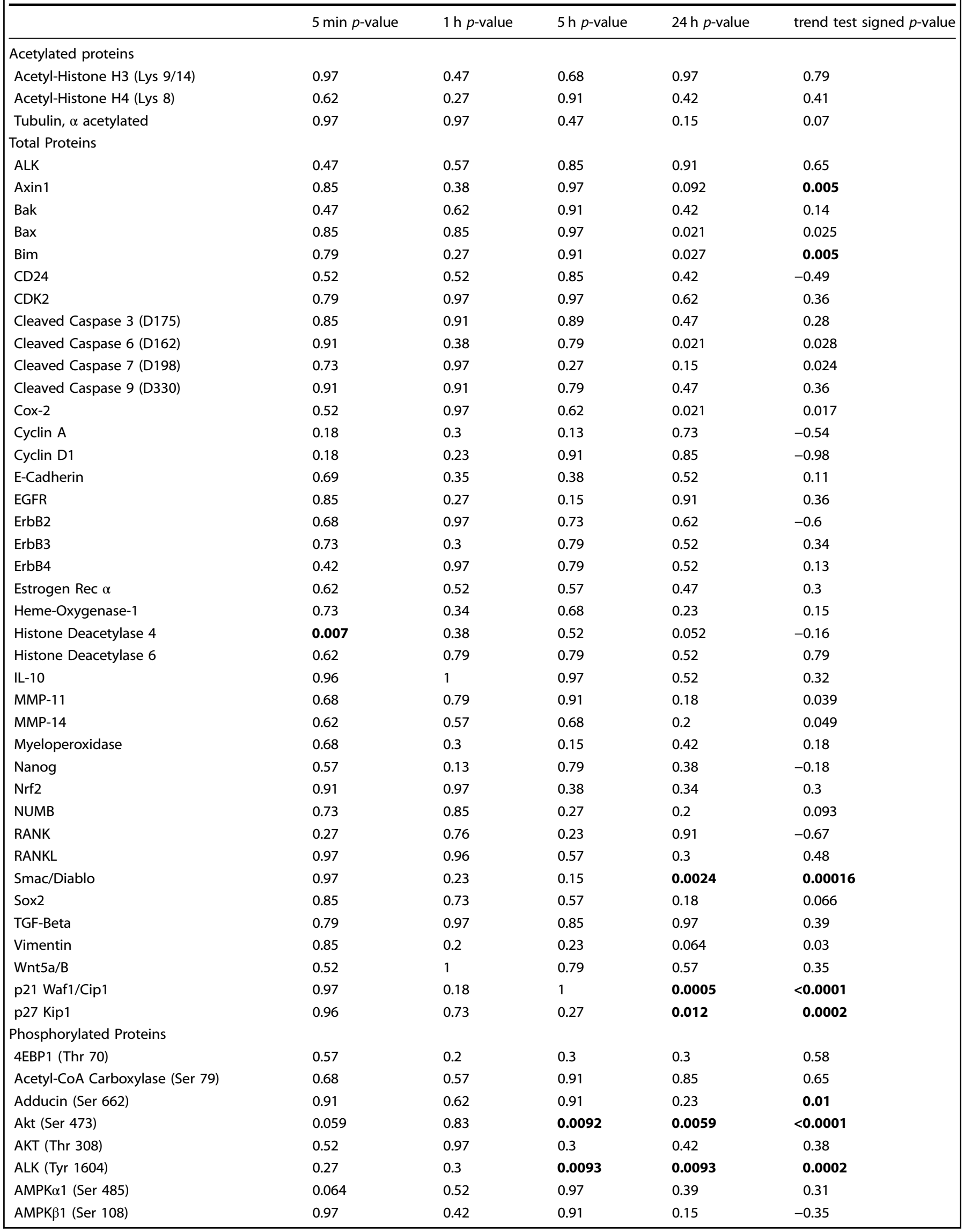


17Pj Mapping Prote in Activation in UV Exposed Skin

\begin{tabular}{|c|c|c|c|c|c|}
\hline & $5 \min p$-value & $1 \mathrm{~h} p$-value & $5 \mathrm{~h} p$-value & $24 \mathrm{~h} p$-value & trend test signed $p$-value \\
\hline ASK1 (Ser 83) & 0.34 & 0.38 & 0.34 & 0.083 & 0.052 \\
\hline ATP-Citrate Lyase (Ser 454) & 0.23 & 0.077 & 0.0049 & 0.0005 & $<0.0001$ \\
\hline Aurora A/B/C (Thr 288/232/198) & 0.47 & 0.52 & 0.62 & 0.47 & 0.81 \\
\hline Bcl-2 (Ser 70) & 0.73 & 0.91 & 0.73 & 0.73 & 0.48 \\
\hline B-Raf (Ser 445) & 0.91 & 0.3 & 0.13 & 0.064 & 0.0033 \\
\hline c-Abl (Thr 735) & 0.42 & 0.97 & 0.85 & 0.68 & -0.7 \\
\hline c-Abl (Tyr 245) & 0.47 & 0.91 & 0.97 & 0.68 & 0.97 \\
\hline c-Kit (Tyr 703) & 0.91 & 0.85 & 0.97 & 0.34 & 0.3 \\
\hline EGFR (Tyr 1068) & 0.48 & 0.023 & 0.0039 & 0.019 & 0.0002 \\
\hline EGFR (Tyr 1173) & 0.97 & 0.57 & 0.2 & 0.042 & 0.0015 \\
\hline EGFR (Tyr 992 & 0.68 & 0.092 & 0.52 & 0.13 & -0.11 \\
\hline elF4G (Ser 1108) & 0.56 & 0.27 & 0.016 & 0.0005 & $<0.0001$ \\
\hline eNOS (Ser 1177) & 0.85 & 0.0093 & 0.00098 & 0.0005 & $<0.0001$ \\
\hline eNOS/NOS III (Ser 116) & 0.79 & 0.73 & 0.34 & 0.021 & 0.0027 \\
\hline ErbB2 (Tyr 1248) & 0.97 & 0.91 & 0.26 & 0.23 & -0.23 \\
\hline ErbB2 (Tyr 877) & 0.52 & 0.42 & 0.13 & 0.31 & 0.13 \\
\hline ErbB3 (Tyr 1289) & 0.34 & 0.0093 & 0.00049 & 0.18 & $<0.0001$ \\
\hline ERK1/2 (Thr 202/Tyr 204) & 0.5 & 0.0087 & 0.034 & 0.0005 & $<0.0001$ \\
\hline Histone H3 (Ser 10) & 1 & 0.79 & 0.62 & 0.064 & 0.031 \\
\hline IGF-1R/IR (Tyr 1135/36/1150/51) & 0.31 & 0.1 & 0.26 & 0.18 & 0.14 \\
\hline $\operatorname{IkB} \alpha(\operatorname{Ser} 32 / 36)$ & 0.62 & 0.79 & 0.38 & 0.27 & 0.15 \\
\hline IRS1 (Ser 612) & 0.79 & 0.23 & 0.2 & 0.0034 & $<0.0001$ \\
\hline Jak1 (Tyr 1022/23) & 1 & 0.85 & 0.79 & 0.27 & 0.16 \\
\hline Lck (Tyr 505) & 0.42 & 0.91 & 0.68 & 0.79 & 0.5 \\
\hline LIMK1/2 (Thr 508/506) & 0.97 & 0.62 & 0.27 & 0.13 & 0.036 \\
\hline LKB1 (Ser 334) & 0.91 & 0.2 & 0.15 & 0.52 & 0.12 \\
\hline LKB1 (Ser 428) & 0.23 & 0.034 & 0.0034 & 0.0005 & $<0.0001$ \\
\hline MDM2 (Ser 166) & 0.73 & 0.052 & 0.0068 & 0.0005 & $<0.0001$ \\
\hline MEK1/2 (Ser 217/221) & 0.79 & 0.11 & 0.23 & 0.0024 & $<0.0001$ \\
\hline Met (Tyr 1234/35) & 0.97 & 0.97 & 0.38 & 1 & 0.73 \\
\hline Mst1/2 (Thr 183/180) & 0.85 & 0.68 & 0.3 & 0.11 & 0.01 \\
\hline mTOR (Ser 2448) & 0.62 & 0.11 & 0.0049 & 0.0005 & $<0.0001$ \\
\hline NFk $\beta$ p65 (Ser 536) & 0.91 & 0.91 & 0.27 & 0.97 & -0.97 \\
\hline p38 MAPK Thr (180/Y182) & 0.47 & 0.016 & 0.0015 & 0.0024 & $<0.0001$ \\
\hline p70 S6 Kinase (Ser 371) & 0.27 & 0.18 & 0.016 & 0.0005 & $<0.0001$ \\
\hline p90RSK (Ser 380) & 0.11 & 0.42 & 0.059 & 0.0005 & $<0.0001$ \\
\hline p90RSK (Thr 359/S363) & 0.13 & 0.052 & 0.012 & 0.0024 & $<0.0001$ \\
\hline PAK1/2 (Thr 423/402) & 0.79 & 0.57 & 0.13 & 0.0093 & $<0.0001$ \\
\hline
\end{tabular}




\begin{tabular}{|c|c|c|c|c|c|}
\hline & $5 \min p$-value & $1 \mathrm{~h} p$-value & $5 \mathrm{~h} p$-value & $24 \mathrm{~h} p$-value & trend test signed $p$-value \\
\hline PKA C (Thr 197) & 0.91 & 0.62 & 0.68 & 0.18 & 0.069 \\
\hline PKCd (Thr 505) & 1 & 0.91 & 0.76 & 0.68 & 0.76 \\
\hline PKC $\lambda \zeta / /$ (Thr 410/403) & 0.73 & 0.97 & 0.47 & 0.79 & 0.72 \\
\hline PRAS40 (Thr 246) & 0.61 & 0.85 & 0.98 & 0.27 & 0.58 \\
\hline PTEN (Ser 380) & 0.23 & 0.11 & 0.077 & 0.13 & 0.069 \\
\hline Ret (Tyr 905) & 0.62 & 0.13 & 0.11 & 0.42 & 0.19 \\
\hline RSK3 (Thr 356/Ser 360) & 0.62 & 0.11 & 0.0093 & 0.0005 & $<0.0001$ \\
\hline SAPK/JNK (Thr 183/Tyr 185) & 0.57 & 0.97 & 0.021 & 0.042 & 0.0034 \\
\hline Smad1 (S/S)/Smad5 (S/S)/Smad8 (S/S) & 0.79 & 0.89 & 0.57 & 0.23 & 0.1 \\
\hline SMAD2 (Ser 465/467) & 0.73 & 0.79 & 0.47 & 0.85 & 0.66 \\
\hline Src Family (Tyr 416) & 0.1 & 0.57 & 0.0068 & 0.69 & 0.092 \\
\hline Src (Tyr 527) & 0.89 & 0.85 & 0.91 & 0.021 & 0.015 \\
\hline Stat3 (Ser 727) & 0.85 & 0.31 & 0.092 & 0.001 & $<0.0001$ \\
\hline Syk (Tyr 525/526) & 0.52 & 0.91 & 0.62 & 0.42 & -0.063 \\
\hline Tuberin/TSC (Tyr 1571) & 0.79 & 0.91 & 1 & 0.42 & 0.54 \\
\hline Vav3 (Tyr 173) & 0.91 & 0.85 & 0.62 & 0.79 & 0.48 \\
\hline VEGFR 2 (Tyr 951) & 0.3 & 0.47 & 0.27 & 0.052 & 0.032 \\
\hline VEGFR2 (Tyr 996) & 0.52 & 0.0068 & 0.012 & 0.79 & 0.049 \\
\hline
\end{tabular}

comparing the change in expression with an independent cohort of subjects exposed to acute SSL. When comparing the RPPA analytes evaluated in both studies a total of 17 proteins were identified to overlap. A $94.1 \%$ agreement in directional change of expression was identified between these two cohorts, strongly supporting the validity of our findings (Table 2).

\section{DISCUSSION}

In the current study, we utilized RPPA, a powerful proteomics platform that provides for the broad-scale and quantitative measurement of the activation/phosphorylation state of dozens to hundreds of proteins at once from a single input sample. We used RPPA to interrogate the activation of more than 100 specific total proteins and phosphoproteins that comprised key signaling "hubs" in known cancer pathways that are causally involved in growth and mitogenesis, survival, motility, apoptosis, autophagy, inflammation and energy metabolism in sun-protected skin after an acute dose of two MED of SSL. We are not aware of any other studies using the RPPA platform for the broad-scale and quantitative measurement of proteins and phosphoproteins in SSL-irradiated skin. While gene expression arrays are commonly utilized to measure changes in the mRNA expression, the measurement of RNA alone lacks critical information such as post-translational modifications such as protein phosphorylation which drive cellular processes and control cell signaling. ${ }^{18}$

While an important novel attribute of our study is the evaluation of acute SSL effects on human subjects in a clinical setting, a key limitation of RPPA is that we are limited by the size and location of the clinical biopsy sample and thus we cannot perform "kinome wide" measurements of every cellular signalling protein as one could do with billions of cells grown in culture. The number of cells that could be captured by LCM of epidermis was $\sim 15,000$ cells, and thus reducing the number of RPPA proteins that we were able to analyse and present here. Consequently, we chose to focus our analysis on key signalling pathways involved in the epidermal UV-response and in tumorigenic processes such as stress, inflammation, survival, energy metabolism, growth, and differentiation. Our protein panel was based on the literature and knowledge of signaling pathway alterations seen in both mouse and human skin after UV irradiation and in the progression of normal skin to cutaneous SCC. Furthermore, while signalling can be regulated by a number of post-translational modification driven events (e.g., glycosylation, acetylation, etc.), we elected to focus on protein phosphorylation, which commonly drives signalling cascades of interest. As for most immunoassays, RPPA requires the availability of well-performing, highly specific antibodies. Our study included 128 proteins to determine whether an acute dose of two MED of SSL to normally sun-protected skin of healthy volunteers would activate the expression of key cell signaling pathways that are also activated or are altered in expression during the progression of normal skin, to sun-damaged skin, to AK, and finally to SCC. Of the 128 key signaling proteins measured, only a subset of activated proteins achieved statistical significance after correcting for multiple comparisons (Table 1).

In our study, $6 \mathrm{~mm}$ skin biopsies from a sun-protected site were split in half with one half fixed in formalin for IHC (published in Bermudez et $\mathrm{al}^{20}$ ) and the other half were snap frozen for RPPA. We found evidence of biochemically linked activation of multiple signaling pathways that included a number of individual members 

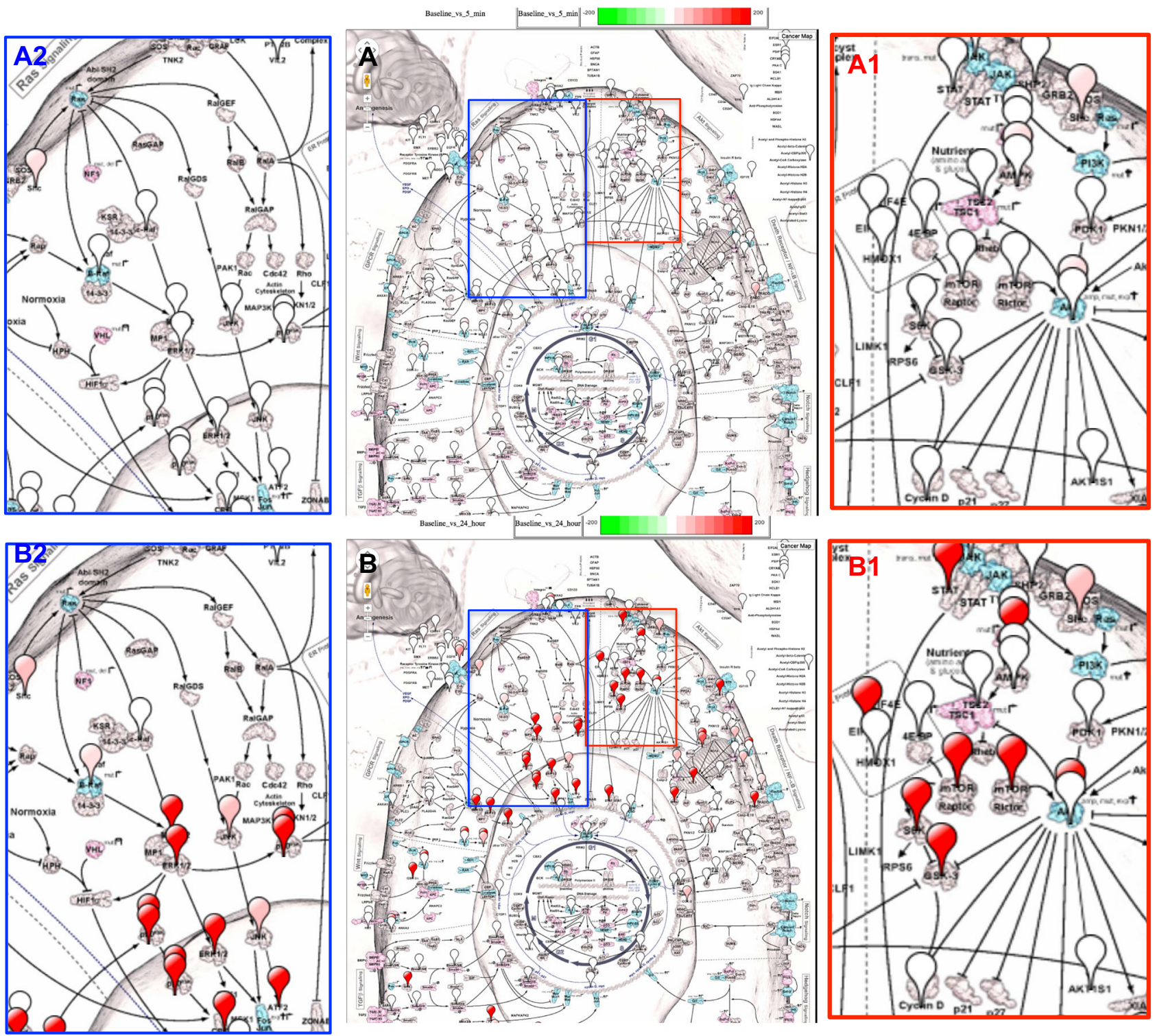

Fig. 1 Protein pathway activation map of the AKT/mTOR and MAPK pathways. Cancer Landscape (CScape) Protein Pathway Activation Maps are shown in the center of the figure comparing the $5 \mathrm{~min}$ compared to baseline $\mathbf{a}$ on top and the $24 \mathrm{~h}$ comparison on the center bottom $\mathbf{b}$. More significant positive differences are shown in increasing shades of red, whereas higher negative differences are shown in green. White balloons represent no significant change. Each balloon pin is placed over the protein measured. Magnified views of the Ras/Raf/MEK/ERK pathway (blue boxes, left top showing the 5 min time point, A1 and bottom right showing the $24 \mathrm{~h}$ time, B1) are shown to reveal pathway detail and the PI3K/AKT/mTOR pathway (red boxes, right top showing the 5 min time point, A2 and bottom left showing the $24 \mathrm{~h}$ time, B2). Images are modified from the "Pathways in Human Cancer" diagram courtesy of Cell Signaling, Inc

of the MAPK pathway, the AKT-mTOR signaling pathways and the cell death/apoptosis-related pathways as well as specific RTK activation such as EGFR, ALK, and vascular endothelium growth factor receptor (VEGFR). There was early and sustained activation of the p38/SAPK/JNK/ERK pathways beginning at $1 \mathrm{~h}$ [i.e., p38 (T180/Y182), ERK1/2 (T202/Y204), MEK1/2 (S217/221), CREB (S133), RSK3 (T356/S360)], while at $5 \mathrm{~h}$ expression of these phosphoproteins/proteins was increased for both the number of proteins expressed and the levels of expression [i.e., SAPK/JNK (T183/Y185), ATF-2 (T71), p90RSK (T359/S363)]. Furthermore, the levels of expression were sustained or even further increased at $24 \mathrm{~h}$. Similarly, activation of the AKT-mTOR pathways was generally increased beginning at the 1 -hr time point with modest additional increases in phosphorylation of GSK3a/ $\beta$ (S21/9) and p70 S6 Kinase (S371). Furthermore, at 5 and $24 \mathrm{~h}$ phosphorylation of AKT
(S473) and mTOR (S2448) were statistically significantly increased. Phospho-elF4G (S1108) was significantly increased at only $24 \mathrm{~h}$. With the exception of FADD (S194), apoptotic proteins were not evident at $5 \mathrm{~min}$, but at $1 \mathrm{~h}$, ATP-Citrate Lyase (S454), BAD (S112), and BAD (S155) become evident at 5 and $24 \mathrm{~h}$. ATP-Citrate Lyase (S454), BAD (S112), and BAD (S155) were further increased. Some additional proteins become evident and others increased at $24 \mathrm{~h}$ (Cleaved Caspase 6, Smac/Diablo, and Bax). We used two independent pathway visualization and network connectivity tools (Figs. 1 and 2), both of which pointed to systemic pathway-based signaling activation of the MEK-ERK, AKT-mTOR, and selected RTK activation.

In a recent publication, ${ }^{20}$ we utilized the formalin-fixed portion of the biopsy from our current study to determine whether SSL would activate the expression of key proteins/phosphoproteins 
Table 2. RPPA analytes compared to an independent cohort

\begin{tabular}{|c|c|c|c|c|}
\hline & $24 \mathrm{~h} p$-value & ssl direction & bse direction & agree/disagree \\
\hline Smac/Diablo & 0.0024 & + & no match & \\
\hline p27 Kip + & $0.0+2$ & + & no match & \\
\hline \multicolumn{5}{|l|}{ Phosphorylated Proteins } \\
\hline ATF-2 (Thr 71) & 0.0039 & + & no match & \\
\hline ATP-Citrate Lyase (Ser 454) & 0.0005 & + & no match & \\
\hline BAD (Ser 112) & 0.0005 & + & + & agree \\
\hline BAD (Ser 155) & 0.0068 & + & + & agree \\
\hline CREB (Ser 133) & 0.0024 & + & + & agree \\
\hline FADD (Ser 194) & 0.0005 & + & no match & \\
\hline GSK-3 $\alpha / \beta$ Ser $(21 / 9)$ & 0.001 & + & + & agree \\
\hline IRS1 (Ser 612) & 0.0034 & + & no match & \\
\hline LKB1 (Ser 428) & 0.0005 & + & + & agree \\
\hline MDM2 (Ser 166) & 0.0005 & + & + & agree \\
\hline MEK1/2 (Ser 217/221) & 0.0024 & + & + & agree \\
\hline mTOR (Ser 2448) & 0.0005 & + & no match & \\
\hline p38 MAPK Thr (180/Y182) & 0.0024 & + & + & agree \\
\hline p70 S6 Kinase (Ser 371) & 0.0005 & + & + & agree \\
\hline p90RSK (Ser 380) & 0.0005 & + & + & agree \\
\hline
\end{tabular}

within the PI3K/Akt/mTOR and MAPK signaling pathways using IHC. Our IHC assays are quantified using image analysis to give a result as the percent positive area of the epidermis to yield data as a continuous variable. Findings from these IHC analyses generally support our RPPA findings herein. Results included a significant but modest increase in AKT (S473) at $5 \mathrm{~h}$ and a significant and sustained increase in mTOR (S2448) over $24 \mathrm{~h}$. We did not measure the same phosphorylation site of 4E-BP1 (T37/46) between IHC and RPPA but found a significant increase at $24 \mathrm{~h}$ through both methodologies. Phospho-p38 (T180/Y182) peaked between 1 and $5 \mathrm{~h}$, and this level was sustained up to $24 \mathrm{~h}$. Expression of phospho-ERK1/2 (T202/Y204) was evident at 5 min and was then increased and sustained to $24 \mathrm{~h}$. Several proteins like COX-2 and cleaved caspase three were significantly increased by IHC; although in some cases RPPA also indicated that levels were increased, the difference was not statistically significant. In another study from our group in which we used four MED of UV in 23 volunteers, we found activation of proteins within the MAPK, PI-3K, p53 and JNK pathways via IHC. ${ }^{15}$ At four MED, we also found significant changes in epidermal expression of phospho-CREB and phospho-GSK-3 $\beta$ from $30 \mathrm{~min}$ to $24 \mathrm{~h}$. The power to detect the change in any expression level from baseline to $24 \mathrm{~h}$ post exposure depends on both the strength of the effect and also on the total number of tests performed (which is equal to the total number of markers/analytes being tested). Since the RPPA analysis considers a much larger number of analytes simultaneously compared to the IHC analysis, the power of the two approaches cannot be directly compared.

Since skin is exposed daily to low dose solar radiation, we believe that the investigation of signaling pathway alterations that result from acute exposures may be relevant to those changes that are seen during skin carcinogenesis. UV is responsible for both initiation and promotion phases of carcinogenesis (a "complete" carcinogen), and chronic exposure to UV results in an accumulation of the critical gene mutations required to drive cells to malignancy; but UV can also act during the promotion phase of carcinogenesis, which could manifest as altered cell signaling ultimately leading to the development of SCC. Interestingly, Martincorena et al. ${ }^{21}$ recently reported that in normal aged sun-exposed eyelid skin there were multiple cancer genes under strong positive selection, including most of the key drivers of cutaneous SCC. They concluded that aged sun-exposed skin was in reality a patchwork of thousands of evolving clones, and while a 

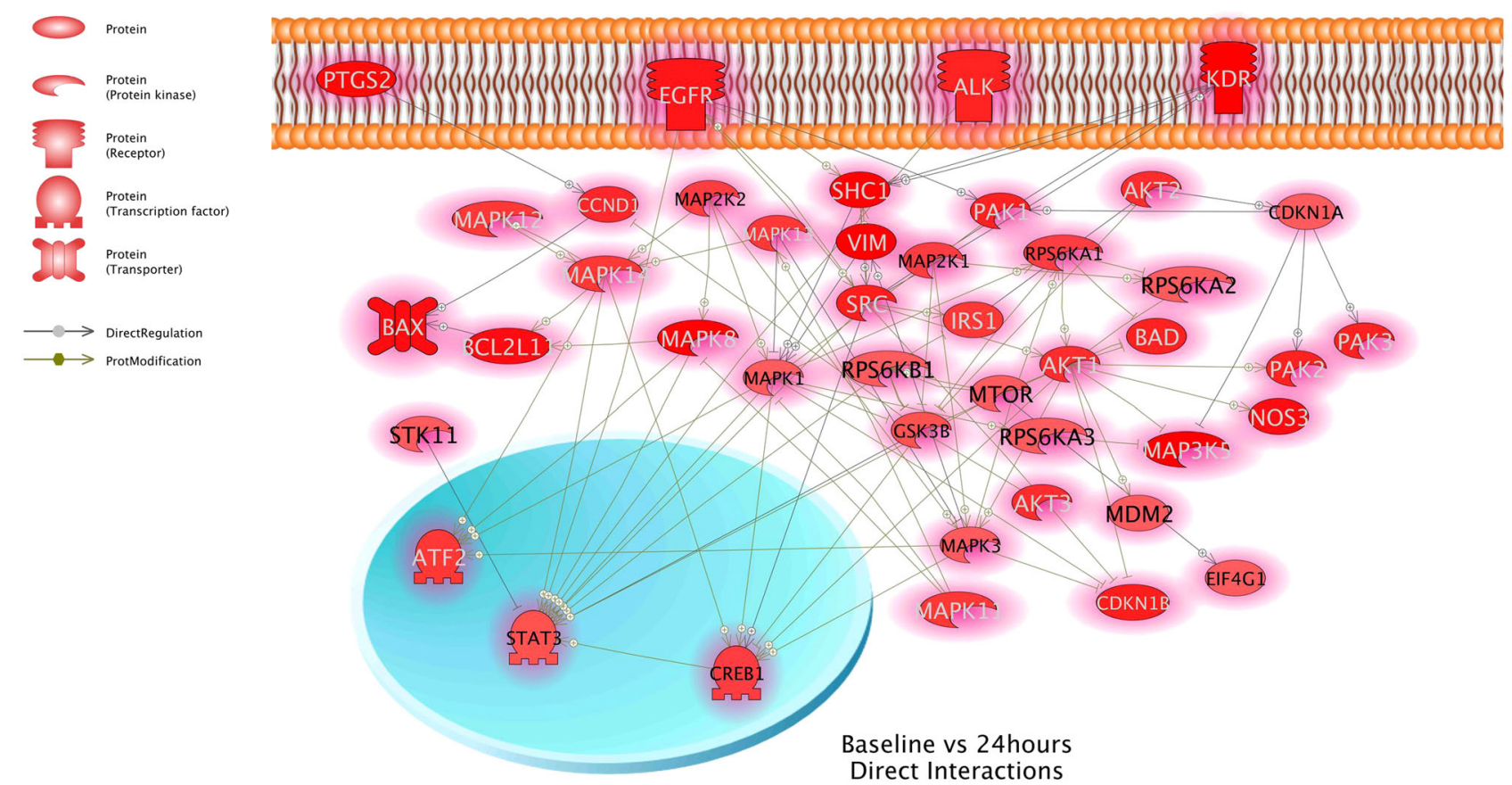

Fig. 2 Protein network analysis of two MED SSL effects on normal sun protected skin at $24 \mathrm{~h}$. Protein network analysis was also conducted using the statistically significant proteins from the RPPA data. Only the statistically significant proteins and phosphoproteins within each time point were used for this analysis

significant number of these clones contained cancer-causing mutations, the tissue but was able to maintain normal histology.

Using RPPA, we previously ${ }^{22}$ found statistically significant differences in protein expression and activation within the MEKERKs and Akt/mTOR signaling pathways in AK compared to SCC and normal skin. ${ }^{22}$ Of interest to the current study, we found that the phosphorylated forms of p38 (T180/Y182), ERK1/2 (T202/ Y204), MEK1/2 (S217/221), mTOR (S2448), elF4G (S1108), p70S6K1 (S371), 4EBP1 (T70), and GSK-3a/ $\beta$ (S21/9), were significantly increased in SCC compared to AK. Furthermore, phospho-4EBP1 (S65) and phospho-Akt (S473) were increased in AK compared to normal skin. ${ }^{22}$, 23 Our broader pathway activation mapping analysis performed and described herein provide the most comprehensive signaling analysis ever performed for UV exposure effects. Our analysis confirmed these previous findings ${ }^{23}$ and also implicated activation of other signaling architecture such as receptor tyrosine kinases EGFR, ALK, and VEGFR amongst other proteins described in Table 1.

IHC and RPPA provide complementary assessment of proteins involved in pathways of interest and validation on results when endpoints are shared across both methodologies. In our current study, samples for RPPA were subjected to LCM to highly enrich for the epithelial cells of interest. However, it is still not possible to determine which cells within the epidermis are expressing a particular protein because cells are lysed. In contrast, in situ measurement of proteins by $\mathrm{IHC}$ and image analysis can be fairly easily ascertained in most fixed samples.

We propose that the study of the acute effects of UV on normal skin may serve as a model for the investigation of the effects of SSL on the complex array of signal transduction pathways. It is likely that at least some of these pathways are involved in chronic or UV-induced carcinogenesis. The study of both acute and chronic solar light exposure may also serve as a model whereby critical signaling pathways and individual proteins can be identified as potential biomarkers for use as endpoints in clinical trials or as companion biomarkers. Moreover, the study of both acute and chronic solar light exposure may allow for the development of interventions such as targeted therapies in a much shorter amount of time than required for studies using cancer as an endpoint.

\section{MATERIALS AND METHODS}

Study population

Study participants were recruited from a pool of subjects who had been previously screened and/or participated in previous skin chemoprevention trials and had agreed to be re-contacted for future studies. The eligibility criteria for participants from this pool included age of 18 years or older and Fitzpatrick skin types II (burns easily, tans poorly) or III (burns moderately, tans gradually). Exclusion criteria included immunosuppression, serious concurrent illness, invasive cancer (including any type of skin cancer) within the past 5 years and baseline serum chemistry values outside of normal limits. In addition, those using photosensitizing medications or topical medications on the test area during the past 30 days were ineligible. Individuals taking mega doses of vitamins were not eligible (i.e., more than five times the RDA, more than five capsules of multivitamins, $400 \mathrm{IU}$ of vitamin E, $200 \mathrm{~g}$ of selenium, and $1 \mathrm{~g}$ of vitamin C). Additional exclusion criteria included individuals with a history of sun exposure to the buttocks within 30 days of randomization and participants must have agreed to avoid sun exposure during the study period. Finally, individuals with a known allergy to lidocaine were ineligible. The University of Arizona Institutional Review Board approved the study and written informed consent was obtained from all study participants. Methods were performed in accordance with relevant regulations and guidelines.

\section{Administration of solar simulated light}

Minimal erythema dose (MED). The MED of SSL was determined for each individual using a Multiport UV Solar Simulator Model 600 (Solar Light Co., Philadelphia, PA, USA) and corroborated by using a reflectance spectrophotometer (Minolta Chroma Meter Model CR-200; Minolta Corporation, Osaka, Japan). The spectrum of light generated by the Solar Simulator consisted of $8.7 \%$ UVB and $91.3 \%$ UVA. ${ }^{19}$ The dose of emission was precisely regulated to be limited to UVA and UVB spectra $(290-390 \mathrm{~nm})$. MED was defined as the smallest dose of energy necessary to produce confluent erythema with four distinct borders at $24 \mathrm{~h}$ post-exposure. MED 
was determined on a buttock area previously unexposed to sunlight. Each test area was subdivided into six subsites (each $1 \mathrm{~cm}^{2}$ ) corresponding to the liquid light guide pattern on the solar simulator. The solar simulator was calibrated prior to each use and a series of six increasing SSL radiation exposures were administered to each subsite area. Following exposure, the test sites were covered until evaluations were completed. ${ }^{20}$

Administration of $2 X$ MED. After determination of the MED for each individual, the contralateral buttock was exposed to 2 times the MED. A 4 $\mathrm{mm}$ skin punch biopsy sample was collected from one buttock at baseline prior to SSL exposure and additional $4 \mathrm{~mm}$ punch biopsies were removed at $5 \mathrm{~min}, 1,5$, and $24 \mathrm{~h}$ post-SSL irradiation. Biopsy sites were then sutured and subjects returned to the clinic for suture removal at $\sim 1$ week.

\section{Reverse phase protein array}

Highly enriched populations of $\sim 6000$ epithelial cells were obtained from skin samples by UV cutting laser microdissection using the Veritas (Molecular Devices, Sunnyvale, CA, USA). Microdissected cells were lysed on the CapSure Macro LCM Caps (Molecular Devices, Sunnyvale, CA, USA) using with a 1:1 mixture of T-Per ${ }^{\mathrm{TM}}$ Tissue Protein Extraction Reagent (Pierce, Rockford, IL, USA) and 2X Tris-Glycine SDS Sample Buffer (Invitrogen, Carlsbad, CA, USA) containing 5\% B-mercaptoethanol. Reverse Phase Protein Microarrays were prepared using an Aushon 2470 solid pin microarrayer (Aushon Biosystems, Billerca, MA, USA). A series of positive control lysates consisting of cell lines treated with compounds that cause broad phosphoprotein increases (e.g., pervanadate, calyculin) or negative controls (untreated cells) were also printed and slides were stored desiccated at $-20^{\circ} \mathrm{C}$ prior to staining with antibody. For estimation of total protein amounts, selected arrays were stained with Sypro Ruby Protein Blot Stain (Invitrogen, Carlsbad, CA, USA) according to the manufacturer's instructions and visualized on the NovaRay scanner (Alpha Innotech, San Leandro, CA, USA). Printed slides were prepared for staining by treating with $1 x$ Reblot (Chemicon, Temecula, CA, USA) for $15 \mathrm{~min}$, followed by $2 \times 5 \mathrm{~min}$ washes with PBS. Slides were treated for at least $5 \mathrm{~h}$ or overnight with blocking solution $(1 \mathrm{~g}$ l-block; Applied Biosystems, Bedford, MA, USA), $0.5 \%$ Tween-20 in $500 \mathrm{~mL}$ PBS) with constant rocking. Blocked arrays were stained with antibodies on an automated slide stainer (Dako North America, Inc., Carpinteria, CA, USA) using the Catalyzed Signal Amplification System kit according to the manufacturer's recommendation (CSA; Dako). Briefly, endogenous biotin was blocked for $10 \mathrm{~min}$ with the biotin blocking kit (Dako), followed by application of protein block for 5 min; primary antibodies were diluted in antibody diluent and incubated on slides for $\mathbf{3 0 ~ m i n}$ and biotinylated secondary antibodies were incubated for $15 \mathrm{~min}$. Signal amplification involved incubation with a streptavidin-biotinperoxidase complex provided in the CSA kit for $15 \mathrm{~min}$, and amplification reagent (biotinyl-tyramide/hydrogen peroxide, streptavidin-peroxidase) for $15 \mathrm{~min}$ each. A signal was generated using streptavidin-conjugated IRDye680 (LI-COR Biosciences, Lincoln, NE, USA). Slides were allowed to air dry following development. All antibodies were subjected to extensive validation for single band, appropriate MW specificity by Western blot, as well as phosphorylation specificity through the use of cell lysate controls (e.g.. HeLa cells $+/-$ pervanadate, Jurkat cells $+/-$ Calyculin purchased from Cell Signaling as lysates). The RPPA community has developed best practices and conventions for antibody validation. ${ }^{24}$ These include single band validation on Western blot, as well as the use of positive and negative controls that are printed on the same array as the experimental samples. These methods have been used by our lab and reported in over 150 peer-reviewed publications to date. Indeed, in early publications when key RPPA findings were evaluated by Western blot, all findings were successfully validated. ${ }^{25}$ Importantly, RPPA findings have also been crossvalidated using FDA-approved means of measuring the same protein such as parallel IHC and FISH evaluation of HER2 expression. ${ }^{26}$ In this publication, the RPPA findings showed 95 and $97 \%$ concordance with IHC Herceptest ${ }^{\mathrm{TM}}$ and FISH HER2 assays. A full list of all antibodies used in this study along with the vendor and dilution used are shown (Supplemental Table 1).

Stained slides were scanned individually on the NovaRay scanner (Alpha Innotech) or the Vidar Scanner (Vidar Systems, Herndon, VA). The TIF images for antibody-stained slides and Sypro-stained slide images were analyzed using MicroVigene v2.9.9.9 (VigeneTech, Carlisle, MA, USA). Briefly, Microvigene performed spot finding, local background subtraction, replicate averaging and total protein normalization, producing a single value for each sample at each endpoint. All data was background subtracted (local and slide average), normalized to total protein, and all signal values produced for data analysis were at least two standard deviations above background.

\section{Data and statistical analysis}

A total of 128 RPPA proteins were included in the analysis. Each protein was measured at five time points: baseline (BL), $5 \mathrm{~min}, 1 \mathrm{~h}, 5 \mathrm{~h}$, and $24 \mathrm{~h}$ after exposure to SSL. Supplemental Table 2 contains raw RPPA data for each subject and time point. The distribution of each protein was first examined graphically using histograms and box plots, and the appropriate transformation applied, if necessary, to achieve approximate normality. An overall homogeneity test was first performed to check whether the levels of each protein at the different time points were the same. This test was implemented by fitting a generalized estimating equations (GEE) model for protein values across all time points and performing a multivariate Wald test on estimated parameters from this model. GEE was used to account for within-subject correlation. Due to the large number of proteins evaluated, the Benjamini-Hochberg method was used to adjust for multiple testing with the false discovery rate controlled at the 0.05 level. If the overall homogeneity test for any protein remained significant after the multiple testing adjustment (indicating that the levels of the protein were not the same across the five time points). The Wilcoxon signed-rank test was then conducted for each protein to compare the level at baseline to the levels at $5 \mathrm{~min}, 1 \mathrm{~h}, 5 \mathrm{~h}$, and $24 \mathrm{~h}$ after SSL exposure. Since four pairwise comparisons were made for each protein, the Bonferroni method was used to adjust for multiple comparisons, with significance level set at 0.05 divided by the four timepoints $(p \leq 0.0125)$. In addition, a trend test was also conducted to check whether there was a monotonic trend from baseline to $24 \mathrm{~h}$ after SSL exposure. For this test, a GEE model was fitted for levels of each protein with time points as covariate (coded as numerical values $0,1,2,3$, and 4). As previously indicated, due to the large number of proteins, the Benjamini-Hochberg method was used to adjust for multiple testing, with the false discovery rate controlled at the 0.05 level. We also validated our findings using an independent validation set. For each analyte with significant change $(p<0.0125)$ from baseline to $24 \mathrm{~h}$, analyte in the validation study representing the same proteins is identified, if existent, and the direction of change from baseline to $24 \mathrm{~h}$ post-exposure was compared to the direction of change in this study. The rate of agreement was then estimated.

\section{Pathway mapping visualization and protein network analysis}

Statistically significant data derived from the RPPA analysis was visualized for network linkages as previously described. ${ }^{27}$ Briefly, the "Pathways in Human Cancer" diagram (reproduced courtesy of Cell Signaling, Inc., www. cellsignal.com) along with the Google Maps Application Programming Interface (API) (http://code.google.com/apis/maps) was used to create a dynamic web application called CScape (Cancer Landscape) for visualizing and navigating through the RPPA-generated data.

Protein network analysis was also conducted using the statistically significant proteins from the RPPA analysis [Pathway Studio v.11.0 (Elsevier, Amsterdam, Netherlands)]. The software contains the ResNet Mammal v. 11.0 database with functional relationships and pathways of human, mouse and rat genes derived from over 24,000,000 PubMed abstracts and over 3500000 papers from Elsevier and 3rd party groups. The list of significant proteins and phosphoproteins was first mapped to the Huge Gene Nomenclature Committee (HGNC)-approved gene symbols, which were inputted into the software. For significant proteins and phosphoproteins within each time point, we conducted pathway enrichment analysis based on the Fisher's exact test and a $p$ value cut off of 0.05 . We then generated interaction networks to determine the direct interactions between the significant proteins/phosphoproteins. Each interaction in the network is supported by at least one published reference from the ResNet Mammal v.11.0 database. Figure 2 shows one of these networks used to visualize the RPPA data from human skin biopsies obtained $24 \mathrm{~h}$ post-SSL. Statistical analysis found a series of SSL-induced phosphoprotein and protein activation/expression changes that occurred at $24 \mathrm{~h}$. The results showed significant activation of a number of receptor tyrosine kinases (EGFR, ALK, VEGFR) along with downstream COX2 signaling, mitogenic (MEK-ERK pathway), pro-survival (AKT-mTOR pathway) and apoptosis pathways (Fig. 2)

\section{Data availability statement}

The raw data that support the findings of this study is included Supplemental Table 2. Upon reasonable request further information can 
be requested from the University of Arizona Prevention of Skin Cancer P01 Data Sharing Committee (UACC-SkinPPG@uacc.arizona.edu).

\section{ACKNOWLEDGEMENTS}

Research reported in this publication used the Tissue Acquisition and Cellular/ Molecular Analysis Shared Resource at the University of Arizona Cancer Center. Special thanks to the Innovation Center for Biomedical Informatics (ICBI) at the Georgetown University Medical Center for reviewing the RPPA data analysis. We thank Claudius Mueller at George Mason University who generated the CScape images. This work was supported, in part, by PHS Grants P01 CA027502, K07 CA132956, and P30 CA023074 from the National Cancer Institute, National Institutes of Health. This publication is solely the responsibility of the authors and does not necessarily represent the official views of the National Cancer Institute.

\section{AUTHOR CONTRIBUTIONS}

J.G.E., C.C.L., S.P.S., D.S.A., E.F.P., and J.W., conceived the experiments and analyzed the data with help from E.L.W., S.D., Z.D., and A.M.B. for experiment analysis. V.S.C. helped with RPPA studies. C.H. and K.S. performed statistical analysis. J.G.E. wrote the paper with help from C.C.L. and E.F.P.

\section{ADDITIONAL INFORMATION}

Supplementary Information accompanies the paper on the npj Precision Oncology website (doi:10.1038/s41698-017-0037-7).

Competing interests: Dr. Petricoin and Ms. Valerie Calvert have issued and licensed patents concerning the reverse phase protein array technology and held stock in Avant Diagnostics. All the other authors declare that they have no competing financial interests.

Publisher's note: Springer Nature remains neutral with regard to jurisdictional claims in published maps and institutional affiliations.

\section{REFERENCES}

1. Rogers, H. W. et al. Incidence estimate of nonmelanoma skin cancer in the United States, 2006. Arch. Dermatol. 146, 283-287 (2010).

2. Rosen, T. \& Lebwohl, M. G. Prevalence and awareness of actinic keratosis: barriers and opportunities. J. Am. Acad. Dermatol. 68, S2-S9 (2013).

3. Guy, G. P. Jr., Machlin, S. R., Ekwueme, D. U. \& Yabroff, K. R. Prevalence and costs of skin cancer treatment in the U.S., 2002-2006 and 2007-2011. Am. J. Prev. Med. 48, 183-187 (2015).

4. Harris, R. B., Griffith, K. \& Moon, T. E. Trends in the incidence of nonmelanoma skin cancers in southeastern Arizona, 1985-1996. J. Am. Acad. Dermatol. 45, 528-536 (2001).

5. Gasparro, F. P. Sunscreens, skin photobiology, and skin cancer: the need for UVA protection and evaluation of efficacy. Environ. Health. Perspect. 108, 71-78 (2000).

6. Chen, H., Weng, Q. Y. \& Fisher, D. E. UV signaling pathways within the skin. J. Invest. Dermatol. 134, 2080-2085 (2014).

7. Muthusamy, V. \& Piva, T. J. The UV response of the skin: a review of the MAPK, NFkappaB and TNFalpha signal transduction pathways. Arch. Dermatol. Res. 302, 5-17 (2010).

8. Brash, D. E. UV signature mutations. Photochem. Photobiol. 91, 15-26 (2015).

9. Carr, T. D., DiGiovanni, J., Lynch, C. J. \& Shantz, L. M. Inhibition of mTOR suppresses UVB-induced keratinocyte proliferation and survival. Cancer Prevention Res. 5, 1394-1404 (2012).

10. Cao, C. \& Wan, Y. Parameters of protection against ultraviolet radiation-induced skin cell damage. J. Cell. Physiol. 220, 277-284 (2009).
11. Krutmann, J. Ultraviolet A radiation-induced biological effects in human skin: relevance for photoaging and photodermatosis. J. Dermatol. Sci. 23(Suppl 1), S22-S26 (2000).

12. Bachelor, M. A., Silvers, A. L. \& Bowden, G. T. The role of p38 in UVA-induced cyclooxygenase-2 expression in the human keratinocyte cell line, HaCaT. Oncogene 21, 7092-7099 (2002).

13. Keum, Y. S., Kim, H. G., Bode, A. M., Surh, Y. J. \& Dong, Z. UVB-induced COX-2 expression requires histone $\mathrm{H} 3$ phosphorylation at Ser10 and Ser28. Oncogene 32, 444-452 (2013).

14. Bowden, G. T. Prevention of non-melanoma skin cancer by targeting ultravioletB-light signalling. Nat. Rev. Cancer 4, 23-35 (2004).

15. Einspahr, J. G. et al. Cross-validation of murine UV signal transduction pathways in human skin. Photochem. Photobiol. 84, 463-476 (2008).

16. Lee, J. K., Ko, S. H., Ye, S. K. \& Chung, M. H. 8-Oxo-2'-deoxyguanosine ameliorates UVB-induced skin damage in hairless mice by scavenging reactive oxygen species and inhibiting MMP expression. J. Dermatol. Sci. 70, 49-57 (2013).

17. Strozyk, E. \& Kulms, D. The role of AKT/mTOR pathway in stress response to UVirradiation: implication in skin carcinogenesis by regulation of apoptosis, autophagy and senescence. Int. J. Mol. Sci. 14, 15260-15285 (2013).

18. Rapkiewicz, A. et al. The needle in the haystack: application of breast fine-needle aspirate samples to quantitative protein microarray technology. Cancer 111, 173-184 (2007)

19. Ravnbak, M. H. Objective determination of Fitzpatrick skin type. Dan. Med. Bull. 57, B4153 (2010).

20. Bermudez, Y. et al. Activation of the PI3K/Akt/mTOR and MAPK signaling pathways in response to acute solar-simulated light exposure of human skin. Cancer Prevention Res. 8, 720-728 (2015).

21. Martincorena, I. et al. Tumor evolution. High burden and pervasive positive selection of somatic mutations in normal human skin. Science 348, 880-886 (2015).

22. Einspahr, J. G. et al. Functional protein pathway activation mapping of the progression of normal skin to squamous cell carcinoma. Cancer Prevention Res. 5, 403-413 (2012).

23. Chen, S. J. et al. Activation of the mammalian target of rapamycin signalling pathway in epidermal tumours and its correlation with cyclin-dependent kinase 2. Br. J. Dermatol. 160, 442-445 (2009).

24. Akbani, R. et al. Realizing the promise of reverse phase protein arrays for clinical, translational, and basic research: a workshop report: the RPPA (Reverse Phase Protein Array) society. Mol. Cell. Proteomics 13, 1625-1643 (2014).

25. Silvestri, A. et al. Protein pathway activation mapping of colorectal metastatic progression reveals metastasis-specific network alterations. Clin. Exp. Metastasis 30, 309-316 (2013).

26. Wulfkuhle, J. D. et al. Molecular analysis of HER2 signaling in human breast cancer by functional protein pathway activation mapping. Clin. Cancer Res. 18, 6426-6435 (2012).

27. Einspahr, J. G. et al. Functional protein pathway activation mapping of the progression of normal skin to squamous cell carcinoma. Cancer Prev Res (Phila) $\mathbf{5}$, 403-413 (2012).

Open Access This article is licensed under a Creative Commons Attribution 4.0 International License, which permits use, sharing,
adaptation, distribution and reproduction in any medium or format, as long as you give appropriate credit to the original author(s) and the source, provide a link to the Creative Commons license, and indicate if changes were made. The images or other third party material in this article are included in the article's Creative Commons license, unless indicated otherwise in a credit line to the material. If material is not included in the article's Creative Commons license and your intended use is not permitted by statutory regulation or exceeds the permitted use, you will need to obtain permission directly from the copyright holder. To view a copy of this license, visit http://creativecommons. org/licenses/by/4.0/.

(c) The Author(s) 2017 